\title{
DIAGNÓSTICO Y PUESTA EN MARCHA DE UN PROCESO DE GESTIÓN INTEGRAL DE ACTIVOS EN UNA EMPRESA DE SERVICIOS PETROLEROS
}

\author{
Enrique Daniel Sanmarco (DISIM/UNLP, Argentina) enrique.sanmarco@ing.unlp.edu.ar
}

\begin{abstract}
Resumo: En este trabajo se pretende mostrar una metodología desarrollada y aplicada para evaluar la Gestión Integral de los Activos de una empresa, en este caso orientada a los servicios, a partir de un diagnóstico de situación temporal, con la participación activa de los integrantes de la misma, basada en indicadores internacionalmente aceptados, para proponer un plan de acción basado en la visión y misión que tiene la empresa, con el propósito de generar un plan estratégico orientado a la mejora continua y al posicionamiento de la misma en lugar similar o mejor frente a las empresas que prestan los mismos servicios. En el trabajo se propone a la Gestión Integral de los Activos como un factor superador del Mantenimiento de equipos o instalaciones.
\end{abstract}

Palabras clave: Gestión integral de activos. Proceso. Tácticas de mantenimiento. Autoevaluación. Estrategia.

\section{Introducción}

La Gestión Integral de los Activos de una empresa requiere focalizar la atención en todos aquellos factores que tienen que ver con los mismos, desde el recurso humano, esencial en la misma, el conocimiento tecnológico del equipamiento sobre el cual se va a trabajar, los medios y las herramientas con que se va interactuar, el entorno en que se encuentran, pero por sobre todo, mantener un orden que no pierda la visión de los objetivos a lograr.

Sobre estos supuestos y, conforme a referentes normativos e indicadores de gestión reconocidos internacionalmente en la Industria Petrolera y afines, se trata de encontrar un ordenamiento lógico y secuencial del presente traba- jo, utilizando una metodología práctica, que fue la propuesta y realizada durante el tiempo en que se llevó a cabo el trabajo que se presenta, donde se incluyó la preparación de información para un autodiagnóstico, su evaluación, presentación a los Gerentes de un estado de situación vinculada a la autoevaluación, recopilación de la información posterior para elaborar acciones, identificación in situ de funcionamiento de servicios que se prestaban, para finalmente hacer una propuesta de acción con el propósito de generar un proceso de cambios estratégicos que permitieran alcanzar las metas que se deseaban.

Se propuso un objetivo en el corto plazo que fue: "Mostrar a los clientes una gestión ordenada y en proceso de mejora continua" 
Dentro de los alcances que se establecieron figuraban el definir, documentar y comunicar la Visión y Misión de la Empresa a los clientes en los servicios que se prestaban, según normas internacionalmente reconocidas.

Además se pretendía conocer en detalle la situación actual del funcionamiento de la Gestión. Establecer un mapa con el estado de situación en los diversos componentes de la gestión de la operación y mantenimiento. Definir un modelo de Gestión de Operación y Mantenimiento sobre el cual trabajar y poder hacer permanentes comparaciones en función de indicadores reconocidos, con objetivos medibles y reconocidos. Aprovechar todo el sistema de Gestión actual, sus procedimientos ordenándolos en función de la Visión y Misión definidas.

También se pretendía preparar dos líderes coordinadores para el seguimiento de implementaciones de mediano plazo.

Dado el tipo de industria que se atendía, trabajar sobre la implementación de la NORMA ISO 14224 para que sea soporte del modelo de codificación de equipos, situaciones y permita, registrar, ordenar y establecer un lenguaje común para todos los clientes y servicios que se prestan.

\section{Grupos Focalizados}

Si bien la el grupo focalizado es una Empresa de servicios del sector de los servicios de la industria petrolera e hidrocarburos, se considera que la metodología empleada se puede utilizar en empresas de bienes y servicios adaptando el contenido del trabajo a la misma.

\section{Contexto Histórico}

La Gestión Integral de Activos aplicados a las Empresas y Organizaciones de productoras de bienes y servicios, es una visión que aparece en las últimas décadas a partir de la integración de los procesos industriales con todas las áreas de la organización.

Así no se habla de Mantenimiento como una disciplina independiente del proceso productivo si no por el contrario integrado al mismo. Lo mismo ocurre con la Ingeniería del diseño y otras disciplinas que anteriormente se consideraban como sectores independientes y mal llamados de servicios.

La aparición de las Normas como factor comparativo y la orientación de las organizaciones a la mejora continua hizo que esta visión cambiara y hoy, se puede decir sin temor a equivocarse, que toda empresa u organización que se precie de competir en el mercado debe tener incluida en su estrategia de negocios esta visión.

La Gestión de Activos puede definirse como:

"Las actividades y prácticas coordinadas y sistemáticas a través de las cuales una organización maneja de manera óptima y sostenible sus activos y sistemas de activos, su desempeño, riesgos y gastos asociados durante su ciclo de vida con el propósito de lograr el plan estratégico organizacional" (PAS 55-1:2008).

Podemos concluir que la visión del siglo XXI, requiere que cada empresa y/u organización se enmarque en este contexto como la razón de su subsistencia. 


\section{Ventajas y desventajas del trabajo propuesto}

La investigación y trabajos realizados tienen su fundamento en lo expresado en los puntos anteriores.

Como ventaja sustancial se destaca que la metodología aplicada, el orden propuesto que permite recorrer a una empresa en sentido vertical pero también horizontal, permitiendo tener una visión integral de la misma con la activa participación de sus integrantes.

Por otro lado la utilización de normativas vigentes internacionalmente no deja de ser una ventaja.

Como desventaja no se puede soslayar que este tipo de investigaciones y trabajos tiene una componente subjetiva que puede distorsionar algunos resultados pero que en el fondo no implican descalificar las conclusiones a las que se puede arribar y la propuesta de acción

\section{Etapas de realización del trabajo}

\subsection{Planeamiento}

Se comenzó con el Autodiagnóstico, proceso que permitió, sobre la base de diversos elementos planteados como referentes y utilizando una metodología de encuesta anónima, multisectorial y multifuncional, conocer como es referenciada por los integrantes de la empresa la gestión de los activos y el grado de conocimiento de su importancia en el funcionamiento de la misma.

En el análisis previo se fijaron varios ejes que actuaron como referentes y que trataron de individualizar las áreas que se deberían atender en mayor o menor escala para posicionar a la Empresa en una perspectiva no solo competitiva en precio unitario sino Internacionalmente reconocida, con Procesos e Indicadores que se pueda mostrar y sustentar y, por sobre todo, orientada en sus procesos a la Mejora Continua para mostrar los factores diferenciales frente a sus competidores.

Esos ejes fueron:

- Estrategia de Mantenimiento.

- Organización / Recursos Humanos.

- Gestión potencial.

- Tácticas de Mantenimiento.

- Análisis de confiabilidad.

- Medidas de desempeño y Benchmarking.

- Tecnología de información.

- Planificación y Programación de tareas.

- Administración de repuestos y materiales.

- Reingeniería de procesos de Mantenimiento.

Se elaboraron encuestas estructuradas que fueron distribuidas entre los distintos integrantes de la empresa desde los más encumbrados hasta los de menor jerarquía.

Con el resultado de las mismas se elaboró un estado de situación que se definió como autodiagnóstico y se volcaron los resultados en un diagrama tipo "radar".

Los alcances que se quieran lograr en cada caso deberán estar relacionados con la definición del Plan estratégico que se quiera realizar, aunque ya hay algunos ejes que marcaban 


\section{ReLAInEP}

oportunidad de mejoras o al menos zonas potenciales de trabajo.

Cada uno de los ejes se fue desarrollando con encuestas dirigidas, que se vinculaban con la opinión de los miembros integrantes de la empresa.

Para ello se utilizó el siguiente formulario el que fue entregado a los distintos miembros de la Empresa con las indicaciones que figuran en el mismo.

\subsection{Material entregado para la realiza- ción de la investigación}

\section{Formulario (entregado)}

\section{Leer primero las Instrucciones:}

Usted debe ubicarse en la posición que ocupa en la Empresa, independientemente de su nombre y apellido o su número de legajo, tratando de ser lo más fiel con Usted mismo en cada uno de los pasos o preguntas a responder. La escala que hemos adoptado es totalmente arbitraria y solo representa lo que Usted cree que es correcto responder.

Solo vamos a requerirle que lo haga todo en una sola vez y que nos indique UNICAMENTE los datos que le solicitamos (Cuadro 1).

Ubicación primaria de la Persona que responde:

Planta:

División/Departamento: (marcar con una cruz lo que corresponda)

Mantenimiento (M)

Producción (P)

Ingeniería (I)

Almacén/Compras (AC)

Servicios (SE)

Otros (OT)

Puesto Genérico: (marcar con una cruz lo que corresponda)

Gerencia $(G)$

Superintendencia (SI)

Supervisión (SV)

Técnico (T)

Operario $(\mathrm{O})$ 
Administrativo (A)

Los resultados de esta evaluación no serán divulgados sin la aprobación por escrito de la empresa evaluada

\section{CUADRO 1: CANTIDAD DE PERSONAL QUE DEBE RESPONDER}

\begin{tabular}{|c|l|l|}
\hline Departamento & \multicolumn{1}{|c|}{ Puestos } & \multicolumn{1}{c|}{ ¿Cuantos? } \\
\hline Producción & Gerentes & Todos los posibles \\
& Superintendentes & Todos los posibles \\
& Supervisores & 2 por cada área (min.) \\
& Técnicos y Operarios & 2 por cada área (min.) \\
& Administrativos & \\
& & \\
\hline $\begin{array}{c}\text { Mantenimiento e Inge- } \\
\text { niería }\end{array}$ & Gerentes & Todos los posibles \\
& Superintendentes & Todos los posibles \\
& Supervisores & 2 por cada área (min.) \\
& Técnicos y Operarios & 2 por cada área (min.) \\
& Administrativos & 1 por cada área (min.) \\
\hline Almacenes, Compras, & Gerentes & Todos los posibles \\
Servicios y Otros & Superintendentes & Todos los posibles \\
& Supervisores & 1 por cada área (min.) \\
& Técnicos y Operarios & 1 por cada área (min.) \\
& Administrativos & 1 por cada área (min.) \\
\hline
\end{tabular}

Nota1: en todos los casos se debe asignar a la palabra ACTIVO el significado de EquiposInstalaciones-Sistemas.

Nota2: Usted deberá asignar un puntaje de acuerdo a la escala de abajo considerando que piensa es más acorde a su criterio personal (Tabla 1).

Nota: No se preocupe en sumar los puntos que será realizado por nosotros al final de todas las encuestas. Esta encuesta se realiza al solo efecto de tener un perfil de la situación actual vista por los mismos integrantes de la Empresa

\section{TABLA 1: PUNTAJE DE LA ESCALA}

\begin{tabular}{ll}
\hline & Puntaje \\
\hline Estoy Totalmente de acuerdo & 4 \\
Estoy Mayormente de acuerdo & 3 \\
Estoy Algo de acuerdo & 2 \\
Estoy en Desacuerdo & 1 \\
No conozco & 0 \\
\hline
\end{tabular}

y no lo compromete absolutamente en nada.

Solo queremos conocer su punto de vista para elaborar una gestión en conjunto.

\section{¡GRACIAS POR SUS APORTES!}

1. Estrategia de mantenimiento (Cuadro 2)

\begin{tabular}{|c|c|}
\hline \multicolumn{1}{|c|}{ Declaración } & $\begin{array}{c}\text { Puntaje } \\
\mathbf{( 4 , 3 , 2 , 1 , 0 )}\end{array}$ \\
\hline $\begin{array}{l}\text { El departamento de mantenimiento tiene una misión y objetivos bien documentados y } \\
\text { comprendidos por el todo el personal de mantenimiento y producción }\end{array}$ & \\
\hline
\end{tabular}




\begin{tabular}{|c|c|}
\hline Declaración & $\begin{array}{c}\text { Puntaje } \\
(4,3,2,1,0)\end{array}$ \\
\hline \multicolumn{2}{|l|}{$\begin{array}{l}\text { La misión y objetivos claramente apoyan la declaración de objetivos y metas de la em- } \\
\text { presa y el papel de mantenimiento para lograr esas metas esta claro. }\end{array}$} \\
\hline \multicolumn{2}{|l|}{$\begin{array}{l}\text { Mantenimiento tiene un plan a largo plazo o estrategia que nos sirve de guía para pro- } \\
\text { yectos de mejoras y que esta de acuerdo con la estrategia de la empresa. }\end{array}$} \\
\hline \multicolumn{2}{|l|}{$\begin{array}{l}\text { Mantenimiento tiene documentados unos principios guía. Mantenimiento es considera- } \\
\text { do como un proceso no como una función. }\end{array}$} \\
\hline \multicolumn{2}{|l|}{$\begin{array}{l}\text { Nuestro enfoque a mantenimiento es proactivo. Tratamos de prevenir todas las paradas } \\
\text { no programadas y cuando una ocurre la reparamos rápidamente. }\end{array}$} \\
\hline \multicolumn{2}{|l|}{$\begin{array}{l}\text { El presupuesto anual de mantenimiento esta basado en un plan de mejoramiento a largo } \\
\text { plazo que incluye restauraciones estratégicamente programadas basándose en el historial } \\
\text { de desempeño del equipo. }\end{array}$} \\
\hline \multicolumn{2}{|l|}{$\begin{array}{l}\text { El presupuesto de mantenimiento esta relacionado con las expectativas de desempeño } \\
\text { del equipo y existen cifras que indican las consecuencias si el trabajo se retrasa. }\end{array}$} \\
\hline $\begin{array}{l}\text { En el presupuesto de mantenimiento se encuentra separado el trabajo de proyectos que } \\
\text { se realizan por el departamento de mantenimiento. En caso contrario, el presupuesto de } \\
\text { proyectos es totalmente separado para identificarlo claramente. }\end{array}$ & \\
\hline
\end{tabular}

\section{Organización/Recursos Humanos (Cuadro 3)}

\begin{tabular}{|c|c|}
\hline Declaración & $\begin{array}{l}\text { Puntaje } \\
4,3,2,1,0\end{array}$ \\
\hline \multicolumn{2}{|l|}{$\begin{array}{l}\text { La cantidad de personal en el departamento de mantenimiento es adecuada, altamente } \\
\text { capacitada y con mucha experiencia. }\end{array}$} \\
\hline \multicolumn{2}{|l|}{$\begin{array}{l}\text { Las necesidades de la planta referentes a mantenimiento están bien definidas y los téc- } \\
\text { nicos saben que se espera de ellos. Los organigramas están actualizados. }\end{array}$} \\
\hline \multicolumn{2}{|l|}{$\begin{array}{l}\text { Mantenimiento esta bastante descentralizado y organizado por área o línea de produc- } \\
\text { ción. }\end{array}$} \\
\hline \multicolumn{2}{|l|}{ Supervisores son responsables por un mínimo de 12 trabajadores de mantenimiento. } \\
\hline \multicolumn{2}{|l|}{$\begin{array}{l}\text { Existe personal administrativo o sistemas que permiten al supervisor pasar } 75 \% \text { de su } \\
\text { tiempo en apoyo directo de su gente. }\end{array}$} \\
\hline \multicolumn{2}{|l|}{ Horas extras representa menos del 5\% del tiempo total de mantenimiento. } \\
\hline \multicolumn{2}{|l|}{$\begin{array}{l}\text { Horas extras no esta concentrado en un oficio, sino bien distribuido entre todo el perso- } \\
\text { nal. }\end{array}$} \\
\hline \multicolumn{2}{|l|}{ Se ofrece formación técnica a todo el personal y es mas de 5 días/año/persona. } \\
\hline \multicolumn{2}{|l|}{$\begin{array}{l}\text { Todos los supervisores de mantenimiento han recibido formación específica para super- } \\
\text { visión. }\end{array}$} \\
\hline \multicolumn{2}{|l|}{ Existe un programa de aprendizaje para formar los técnicos necesarios. } \\
\hline \multirow{2}{*}{\multicolumn{2}{|c|}{$\begin{array}{l}\text { Tenemos estándares establecidos para graduarse del programa de aprendizaje. } \\
\text { Parte de la paga es basada en conocimientos, resultados y productividad. }\end{array}$}} \\
\hline & Parte de la paga es basada en conocimientos, resultados y productividad. \\
\hline \multicolumn{2}{|l|}{$\begin{array}{l}\text { Se utiliza personal contratado para ayudar a nuestra gente durante paradas programadas, } \\
\text { para proyectos o para trabajos especializados. }\end{array}$} \\
\hline & \\
\hline
\end{tabular}

3. Gestión potencial (Cuadro 4)

\begin{tabular}{|l|l|}
\hline \multicolumn{1}{|c|}{ Declaración } & \multicolumn{1}{|c|}{$\begin{array}{c}\text { Puntaje } \\
\mathbf{4 , 3 , 2 , 1 , 0}\end{array}$} \\
\hline $\begin{array}{l}\text { No tenemos un sistema de control absoluto (dictador, organización con procesos de- } \\
\text { masiado rigurosos). }\end{array}$ & \\
\hline $\begin{array}{l}\text { Técnicos de multidisciplinar (e.g.: electricistas que hacen algo de trabajo mecánico o } \\
\text { mecánicos que hacen algo de trabajo eléctrico, etc.) son parte fundamental de nuestra } \\
\text { organización. }\end{array}$ & \\
\hline $\begin{array}{l}\text { Operarios entienden bien el equipo que manejan y se encargan de algunas actividades } \\
\text { de mantenimiento tales como limpieza, lubricación, ajustes y reparaciones pequeñas. }\end{array}$ & \\
\hline $\begin{array}{l}\text { Supervisores generalmente comparten información de desempeño y costos con los } \\
\text { miembros de su equipo. }\end{array}$ & \\
\hline Equipos de mejoramiento continuo están establecidos y funcionan eficazmente. & \\
\hline
\end{tabular}




\begin{tabular}{|l|l|}
\hline \multicolumn{1}{|c|}{ Declaración } & $\begin{array}{c}\text { Puntaje } \\
\mathbf{4 , 3 , 2 , 1 , 0}\end{array}$ \\
\hline $\begin{array}{l}\text { Equipos auto-dirigidos de operarios, técnicos e ingenieros desarrollan la mayor parte } \\
\text { del trabajo. }\end{array}$ & \\
\hline $\begin{array}{l}\text { Mantenimiento siempre esta involucrado en proyectos de expansión, modificaciones, } \\
\text { incluyendo diseño y puesta en marcha de maquinaria. }\end{array}$ & \\
\hline $\begin{array}{l}\text { Técnicos generalmente responden a llamadas de emergencia fuera de las horas de } \\
\text { trabajo. }\end{array}$ & \\
\hline Producción obtiene el apoyo de mantenimiento rápidamente y con poco esfuerzo. & \\
\hline $\begin{array}{l}\text { El técnico de turno se encarga de las emergencias y decide que apoyo necesita sin } \\
\text { consultar con el supervisor. }\end{array}$ & \\
\hline Producción sabe a quién dirigirse en una emergencia. & \\
\hline Tenemos asociaciones establecidas con suministradores y contratistas clave. & \\
\hline Compartir los riesgos forma parte del contrato. & \\
\hline
\end{tabular}

\section{Tácticas de mantenimiento (Cuadro 5)}

\begin{tabular}{|l|l|}
\hline \multicolumn{1}{|c|}{ Declaración } & $\begin{array}{c}\text { Puntaje } \\
\mathbf{4 , 3 , 2 , 1 , 0}\end{array}$ \\
\hline $\begin{array}{l}\text { Menos que 5\% del trabajo total de mantenimiento en horas-hombre es dedicado a } \\
\text { emergencias (e.g.: paradas no programadas). }\end{array}$ & \\
\hline $\begin{array}{l}\text { Mantenimiento basado en condición tiene preferencia sobre mantenimiento basado en } \\
\text { tiempo o ciclos. }\end{array}$ & \\
\hline $\begin{array}{l}\text { Mantenimiento basado en condición, como análisis de vibración, análisis de aceite, y } \\
\text { otros análisis no destructivos y mediciones de desempeño son muy comunes. }\end{array}$ & \\
\hline $\begin{array}{l}\text { Mantenimiento preventivo y predictivo representan 60\% o más del mantenimiento total } \\
\text { en horas-hombre. }\end{array}$ & \\
\hline $\begin{array}{l}\text { Cumplimiento con el programa de MP (mantenimiento preventivo) es alto. 95\% o más } \\
\text { del trabajo de MP se hace como está programado }\end{array}$ & \\
\hline $\begin{array}{l}\text { Los resultados de las inspecciones de MP e historia de paradas se utilizan continua- } \\
\text { mente para mejorar la eficacia del programa de mantenimiento preventivo }\end{array}$ & \\
\hline $\begin{array}{l}\text { Cuando instalamos nuevos equipos, revisamos las recomendaciones de mantenimiento } \\
\text { del fabricante y las modificamos de acuerdo con nuestra operación, entorno y exigen- } \\
\text { cias. }\end{array}$ & \\
\hline $\begin{array}{l}\text { Hemos usado RCM (Reliability Centered Maintenance) (Mantenimiento centrado en la } \\
\text { confiabilidad de equipos) para determinar las tareas de mantenimiento requeridas y si } \\
\text { es así continuamos usando RCM para mejorar el programa de MP. }\end{array}$ & \\
\hline
\end{tabular}

\section{Analisis de confiabilidad (Cuadro 6)}

\begin{tabular}{|l|l|}
\hline \multicolumn{1}{|c|}{ Declaración } & $\begin{array}{c}\text { Puntaje } \\
\mathbf{4 , 3 , 2 , 1 , 0}\end{array}$ \\
\hline $\begin{array}{l}\text { Se mantienen récords o registros del historial de todos los equipos clave indicando } \\
\text { causa de parada y trabajo de reparación. }\end{array}$ & \\
\hline $\begin{array}{l}\text { Paradas de los equipos son analizadas para determinar la causa raíz y mejorar la pre- } \\
\text { vención. }\end{array}$ & $\begin{array}{l}\text { Nuestras medidas preventivas son generalmente exitosas. Podemos, generalmente, } \\
\text { eliminar el problema que nos proponemos sin crear otros nuevos. }\end{array}$ \\
\hline $\begin{array}{l}\text { Tiempo promedio entre paradas (MTBF) y disponibilidad de los equipos son docu- } \\
\text { mentados/calculados/anticipados. }\end{array}$ & \\
\hline Se han hecho estudios de riesgo/valor para optimizar el programa de mantenimiento. & \\
\hline $\begin{array}{l}\text { Todos los equipos han sido clasificados basados en su importancia para producción, y } \\
\text { seguridad personal. }\end{array}$ & \\
\hline $\begin{array}{l}\text { La clasificación se usa para determinar la prioridad de las órdenes de trabajo y a los } \\
\text { proyectos de ingeniería. }\end{array}$ & \\
\hline Damos prioridad a los equipos más críticos. & \\
\hline $\begin{array}{l}\text { Llevamos estadísticas de confiabilidad a pesar de que nuestro personal tiene una buena } \\
\text { idea de que equipo es mejor y peor. }\end{array}$ & \\
\hline RCM (Reliability Centered Maintenance) u otra forma similar de análisis es utilizado & \\
\hline
\end{tabular}




\begin{tabular}{|c|c|}
\hline Declaración & $\begin{array}{r}\text { Puntaje } \\
\mathbf{4 , 3 , 2 , 1 , 0}\end{array}$ \\
\hline para determinar las tareas óptimas de MP (mantenimiento preventivo). & \\
\hline
\end{tabular}

\section{Medidas de desempeño /benchmarking (Cuadro 7)}

\begin{tabular}{|l|l|}
\hline \multicolumn{1}{|c|}{ Declaración } & $\begin{array}{l}\text { Puntaje } \\
\mathbf{4 , 3 , 2 , 1 , 0}\end{array}$ \\
\hline Costos de repuestos y mano de obra son registrados para sistemas claves y equipos. & \\
\hline $\begin{array}{l}\text { Se mantienen récords o registros de paradas no programadas que incluyen las causas } \\
\text { para equipos y sistemas clave. }\end{array}$ & $\begin{array}{l}\text { Estos récords o registros se analizan periódicamente para resolver los problemas } \\
\text { recurrentes }\end{array}$ \\
\hline $\begin{array}{l}\text { El departamento de mantenimiento tiene una serie de indicadores de desempeño que } \\
\text { se miden frecuentemente y se analizan para compararlos con la estrategia de mante- } \\
\text { nimiento y el programa de mejoramiento continuo. }\end{array}$ & $\begin{array}{l}\text { Todo el personal de mantenimiento ha sido adiestrado en el significado de las medi- } \\
\text { das que se usan. }\end{array}$ \\
\hline $\begin{array}{l}\text { La mayor parte del personal puede leer las medidas y determinar si estamos mejoran- } \\
\text { do o no. }\end{array}$ & $\begin{array}{l}\text { Técnicos de mantenimiento en todas las áreas pueden ver la relación entre su trabajo } \\
\text { y el desempeño global del departamento. }\end{array}$ \\
\hline $\begin{array}{l}\text { Si algún oficio / área está mal se puede observar claramente y así corregir la situa- } \\
\text { ción. }\end{array}$ & \\
\hline $\begin{array}{l}\text { Medidas de desempeño son publicadas regularmente y guardadas como referencia a } \\
\text { disposición de todo el personal. }\end{array}$ & \\
\hline $\begin{array}{l}\text { Se usan benchmarks (indicadores comparativos) internos o externos para compara- } \\
\text { ción }\end{array}$ & \\
\hline Tenemos medidas de desempeño de mantenimiento para las 'mejores compañías'. & \\
\hline Estas medidas se usan como objetivos para el departamento. & \\
\hline
\end{tabular}

\section{Tecnología de información (TI) (Cuadro 8)}

\begin{tabular}{|l|l|}
\hline \multicolumn{1}{|c|}{ Declaración } & Puntaje \\
\hline $\begin{array}{l}\text { T,3,2,1,0 } \\
\text { los de repuestos y finanzas. }\end{array}$ & \\
\hline $\begin{array}{l}\text { Nuestro sistema computarizado de mantenimiento es considerado muy valioso y se usa } \\
\text { constantemente tanto para administración de trabajos como de repuestos y materiales. }\end{array}$ & \\
\hline Nuestro sistema computarizado de mantenimiento es fácil de usar. & \\
\hline $\begin{array}{l}\text { La mayor parte del departamento de mantenimiento ha sido instruida en su uso y lo } \\
\text { utilizan con frecuencia. }\end{array}$ & \\
\hline $\begin{array}{l}\text { Nuestros planificadores y programadores de trabajos usan el SCM tanto para planificar } \\
\text { y programar los trabajos como para reservar los repuestos y materiales. }\end{array}$ & \\
\hline $\begin{array}{l}\text { Información de repuestos es fácil de obtener y está conectada con los récords o regis- } \\
\text { tros de los equipos. }\end{array}$ & \\
\hline $\begin{array}{l}\text { Encontrar repuestos para los equipos es fácil y los récords o registros de los repuestos } \\
\text { son generalmente correctos. }\end{array}$ & \\
\hline $\begin{array}{l}\text { Usamos un sistema de administración de proyectos para planificar las paradas progra- } \\
\text { madas, este sistema determina 'camino crítico' y cantidad de recursos humanos por } \\
\text { oficio. }\end{array}$ & \\
\hline $\begin{array}{l}\text { Técnicas de mantenimiento basado en condición son apoyadas por programas automá- } \\
\text { ticos para analizar los datos y proyectar acciones futuras. }\end{array}$ & \\
\hline Sistemas 'Expertos' son utilizados donde se requieren diagnósticos muy complejos. & \\
\hline Considero que el sistema computarizado es una gran ayuda realmente & \\
\hline
\end{tabular}


7. Planificación y programación de trabajos (Cuadro 9)

\begin{tabular}{|c|c|}
\hline Declaración & $\begin{array}{l}\text { Puntaje } \\
\mathbf{4 , 3 , 2 , 1 , 0}\end{array}$ \\
\hline \multicolumn{2}{|l|}{$\begin{array}{l}\text { Existe un registro de todos los equipos de la planta que requieren algún tipo de mante- } \\
\text { nimiento o apoyo de ingeniería durante su ciclo de vida }\end{array}$} \\
\hline \multicolumn{2}{|l|}{$\begin{array}{l}\text { Mas del } 90 \% \text { del trabajo de mantenimiento es iniciado con una orden de trabajo, una } \\
\text { orden de trabajo permanente, una orden de trabajo de MP. o una hoja de inspecciones } \\
\text { de MP. }\end{array}$} \\
\hline \multicolumn{2}{|l|}{$\begin{array}{l}\text { Mas del } 80 \% \text { del trabajo de mantenimiento (preventivo, predictivo y correctivo) son } \\
\text { planificados por un planificador, supervisor u otra persona al menos con } 24 \text { horas de } \\
\text { adelanto, antes de ejecutarlos }\end{array}$} \\
\hline \multicolumn{2}{|l|}{$\begin{array}{l}\text { Ordenes de trabajo que no son de emergencia son investigadas, estimadas y planificadas } \\
\text { (con tareas, materiales y herramientas) por un planificador dedicado a esta función. }\end{array}$} \\
\hline \multicolumn{2}{|l|}{$\begin{array}{l}\text { Se usan estimados de tiempos requeridos para completar tareas con el fin de programar } \\
\text { los recursos humanos adecuadamente. }\end{array}$} \\
\hline \multicolumn{2}{|l|}{ Se utiliza un sistema de prioridad en todas las órdenes de trabajo. } \\
\hline \multicolumn{2}{|l|}{$\begin{array}{l}\text { Prioridades se asignan de acuerdo a un criterio preestablecido y este criterio no se pasa } \\
\text { por alto para hacer lo que queremos en vez de lo que debemos. }\end{array}$} \\
\hline \multicolumn{2}{|l|}{$\begin{array}{l}\text { El programa de trabajo semanal se hace con el asesoramiento de producción y se basa } \\
\text { en un balance entre los requerimientos de producción y la mano de obra disponible de } \\
\text { cada oficio teniendo en cuenta trabajos de emergencia y mantenimiento preventivo. }\end{array}$} \\
\hline \multicolumn{2}{|l|}{$\begin{array}{l}\text { Todas las paradas programadas se administran usando el método de 'camino crítico' u } \\
\text { otro método gráfico que muestra trabajos con la secuencia, recursos y estimados de } \\
\text { tiempo. }\end{array}$} \\
\hline $\begin{array}{l}\text { Trabajo pendiente listo para programar (backlog) es medido y pronosticado para cada } \\
\text { oficio y se mantiene a un máximo de tres semanas para cada oficio. }\end{array}$ & \\
\hline & \\
\hline
\end{tabular}

8. Administración de repuestos y materiales (Cuadro 10)

\begin{tabular}{|l|l|}
\hline \multicolumn{1}{|c|}{ Declaración } & \multicolumn{1}{|c|}{$\begin{array}{c}\text { Puntaje } \\
\mathbf{4 , 3 , 2 , \mathbf { 0 } , 0}\end{array}$} \\
\hline Nivel de servicio de almacén es medido y es generalmente más de 95\%. & \\
\hline $\begin{array}{l}\text { Repuestos agotados (stock outs) representan menos de 3\% de las órdenes del almacén } \\
\text { requeridas por usuarios. }\end{array}$ & \\
\hline Repuestos y materiales están disponibles para su uso cuando se necesitan. & \\
\hline $\begin{array}{l}\text { Se usan almacenes satélite a través de la planta para los artículos más comunes (e.g. } \\
\text { tuercas, tornillos, mangueras, abrazaderas, plomería, artículos eléctricos comunes). }\end{array}$ & \\
\hline $\begin{array}{l}\text { Compra de repuestos y materiales es automática cuando se llega a un nivel preestableci- } \\
\text { do, sin la intervención del personal de mantenimiento. }\end{array}$ & \\
\hline Hay un almacén en un lugar central para herramientas especiales. & \\
\hline $\begin{array}{l}\text { El inventario se revisa regularmente para eliminar repuestos obsoletos o los usados muy } \\
\text { raramente. Se hace un análisis ABC mensualmente. }\end{array}$ & \\
\hline $\begin{array}{l}\text { En situaciones de emergencia, Compras es capaz de encontrar y adquirir repuestos rápi- } \\
\text { damente evitando así paradas no programadas }\end{array}$ & \\
\hline La rotación de inventario es mayor de 1.5 veces/año. & \\
\hline $\begin{array}{l}\text { Niveles de compra y cantidades se basan en tiempo de adquisición, tasa de uso, y des- } \\
\text { cuento por comprar cantidad. }\end{array}$ & \\
\hline Se usa un sistema computarizado para controlar el inventario. \\
\hline El sistema esta totalmente integrado con el de mantenimiento y planificación. & \\
\hline
\end{tabular}


9. Reingeniería de procesos de mantenimiento (Cuadro 11)

\begin{tabular}{|l|l|}
\hline \multicolumn{1}{|c|}{ Declaración } & $\begin{array}{l}\text { Puntaje } \\
\mathbf{4 , 3 , 2 , 1 , 0}\end{array}$ \\
\hline $\begin{array}{l}\text { Procesos clave de mantenimiento (e.g.: planificación, MP) han sido identificados y } \\
\text { documentados 'como son' en diagramas de flujo (mapeados). }\end{array}$ & \\
\hline Estos procesos son un fiel reflejo de como se ejecutan en la actualidad. & \\
\hline $\begin{array}{l}\text { Procesos clave de mantenimiento son revisados y rediseñados regularmente para opti- } \\
\text { mizarlos (e.g. eliminar actividades que no agregan valor). }\end{array}$ & \\
\hline $\begin{array}{l}\text { Los procesos de mantenimiento se actualizan con el sistema computarizado de mante- } \\
\text { nimiento u otro sistema computarizado. }\end{array}$ & \\
\hline $\begin{array}{l}\text { Rediseño de procesos y representación como diagramas de flujo se extiende a adminis- } \\
\text { tración y apoyo técnico. }\end{array}$ & \\
\hline Costos y tiempos de los procesos de mantenimiento se miden regularmente. & \\
\hline Los Costos por actividad están documentados. & \\
\hline
\end{tabular}

\subsection{Ejecución de trabajo planeado}

El desarrollo de cada uno de los ejes fue el siguiente:

\section{a) Estrategia de mantenimiento}

Este punto esta referido claramente a la visualización de los objetivos, vinculados con la misión y visión de la Empresa, el saber porque existe y que quiere lograr, si se vislumbra un plan de acción que les permitiese guiarse en la operación diaria, existiendo una visión proactiva que permita adelantarse a posibles hechos no deseados como roturas o paradas imprevistas.

También refiere a la documentación con que se cuenta, la posibilidad y facilidad de acceso y uso, si existían principios guía documentados $y$, por fin, si el presupuesto con que se manejaba la actividad de servicio a los clientes, era participativo, real y respondía a las necesidades planeadas o se mezclaba con otro tipo de actividades como serían proyectos y/o cambios programados.

Todo esto debería reflejarse en indicadores conocidos por las distintas funciones en las que esta organizado el servicio y con posibilidad de recoger sugerencias y poder tenerlas en cuenta en futuras acciones.

La estrategia del Mantenimiento es solo una parte de la Gestión de Activos y es parte de la Estrategia de la Empresa.

Este eje, como todos los restantes, se evaluó en una escala totalmente arbitraria para tener una referencia con una serie de preguntas en que cada uno debía puntuar de 0 a 4 con estos criterios de elección del puntaje (Tabla 2).

TABLA 2: ESCALA ARBITRARIA

\section{Criterios}

Estoy Totalmente de acuerdo

Estoy Mayormente de acuerdo

Estoy Algo de acuerdo

Estoy en Desacuerdo

No conozco

\begin{tabular}{c} 
Puntaje \\
\hline 4 \\
3 \\
2 \\
1 \\
0 \\
\hline
\end{tabular}




\section{ReLAlnEP}

Sobre un total de 32 puntos máximos, este rubro promedió los 16.25, lo que significaba un calificación promedio de 2 o sea un $50 \%$ del valor máximo.

Desde luego que esto no debe tomarse como algo absoluto, pero esta indicando necesidad de trabajo en esta área o eje, con posibilidades de una mayor participación en la elaboración de las Estrategias y quizás una mayor comunicación dentro de la Empresa en este punto.

Esto se pudo cotejar con algunas observaciones realizadas "in-situ" donde se notó que se tenía muy claro temas como la Seguridad, los Procedimientos de Medio Ambiente y hasta lo que debían cumplir como Normas en los Emprendimientos, pero faltaba un lenguaje común, un mayor conocimiento hacia donde se deben dirigir los esfuerzos, privilegiando la actitud proactiva frente a la reactiva con la que se estaba trabajando.

\section{b) Organización/Recursos Humanos}

Aparece la necesidad de visualizar "como se comprende la Organización del Mantenimiento por el propio personal", las necesidades de las distintas plantas, su vinculación con los RR.HH disponibles, las relaciones de la Supervisión con su personal, su formación para conducirlo, y si la programación de carrera de las personas es ¿vista por el personal? , ¿Se reconoce un plan sobre la persona?, ¿existen estándares determinados y conocidos para ir avanzando?

¿Existe una consideración por capacidad, conocimientos y actitud en el pago?

Este rubro permite avanzar en programas $\mathrm{y}$ delinear carreras, cuadros de reemplazo y definir necesidades, como así también define en que se ocupa el personal propio y que se deriva a terceros.

La autoevaluación sobre un máximo de 56 puntos posibles, con el mismo criterio de calificación empleado anteriormente, promedió en 27.92 puntos equivalentes a un $50 \%$ o sea 2 puntos.

Evidencia un potencial de acción sumamente interesante para poder actuar. Se debe decir que en el contacto con la gente, se pudo ver una actitud en general abierta y hábida de progreso e involucramiento que se debería aprovechar.

\section{c) Gestión Potencial}

En este punto se destaca la visión que se tiene de la actividad de las personas funcionalmente, el tipo de disciplinas unívocas frente a las multidisciplinarias en cuanto a funciones, actividades y conocimientos. El establecimiento de criterios de trabajo en equipo, con auto dirección, con Facilitadores, la orientación a la mejora continua como convencimiento, no solo porque se certifica ISO 9001-2008 por ejemplo, sino porque la organización esta convencida que debe actuar de esa forma en cada cosa que hace, la interrelación entre personal de operación, mantenimiento, servicios y administrativo.

¿Esta el personal involucrado en los riesgos y es consciente de él?

La autoevaluación en esta parte un puntaje de 36.42 sobre 52 posible, lo cual equivale a $65 \%$ del puntaje total lo que muestra un mas que 
interesante observación del potencial por parte del personal.

En este punto se debería aprovechar todo lo que la Empresa ha desarrollado certificando y trabajando activamente con respecto a las normativas ISO y OSHA como se pudo constatar, lo cual muestra una organización dinámica y consustanciada.

Sobre esta base se recomendó algunos puntos orientados no solo mostrar una gestión sino a profundizar lo ya hecho. En este sentido se focalizó en estos aspectos:

1. Establecer un sistema de Procesos básicos sobre los que se apoye toda la estructura de funciones y actividades. Como ejemplo de esto se nombraría: Proceso de planificación, Procesos de recolección de datos e información, Proceso de gestión de materiales; proceso de desarrollo de ingeniería; proceso de gestión de RR.HH. Se está señalando no mas de 6 a 8 PROCESOS que son la columna vertebral sobre los que se apoya el QUE hacer de la Empresa con los Recursos que Invierte y que Producto Final obtiene.

2. Establecer un estudio de desarrollo de carrera en función de lo que se necesita, lo que se tiene y lo que falta. Esto parece importante por la posible expansión de la Empresa en el mediano plazo aunque necesariamente se comprenderá que esta atado a una Planificación Estratégica Clara
3. Trabajar activamente desde el área de RRHH no solo con la Administración de Personal, el Desarrollo de Carrera y la Capacitación sino sobre aspectos como los contemplados en la Norma API 770 de Errores Humanos en todos los niveles no solo como factor correctivo sino como establecimiento de una acción Proactiva.

4. Establecer una Matriz de prioridades sobre lo dicho en los puntos anteriores que derive un Programa de Trabajo en conjunto de las Áreas en el mediano plazo.

\section{d) Tácticas de Mantenimiento}

En este punto se focalizó la atención en el COMO. Es decir la forma en que se realiza la actividad y por lo tanto se diría que acá debería estar lo que se puede denominar KNOW HOW de la Empresa y como se podrán comprender e involucrarse con toda la experiencia y conocimientos adquiridos en las sucesivas actividades, con la participación de todas las áreas, con el estudio de la mejor manera de realizar un trabajo, con los mejores instrumentos y prácticas, con los cuidados sobre la persona y el medio ambiente, con los recursos adecuados y que por sobre todo estableciendo un lenguaje y una forma de obrar común para toda la Empresa.

Esto requiere Análisis, Estudio, Experiencia, Comunicación y Sistematización, y se apoyan necesariamente sobre la base de la columna vertebral que son los Procesos.

En la autoevaluación este rubro alcanzó 20.42 
puntos sobre 32 posibles o sea un $64 \%$ lo cual muestra una buena interpretación de cómo hacer los trabajos.

Cuando se habla de Táctica se refiere a:

1. Objetivo se busca. P/ej: Disponer de documentación calificada para Planificar las tareas, que lleven a maximizar la confiabilidad de operación de los Puentes grúa y aparejos, verificando el cumplimiento del marco legal local y las definiciones de la compañía

2. Alcance que se le da.

3. Equipo de Redacción de Tácticas: quienes intervienen

4. Definiciones, Términos y Abreviaturas: glosario para establecer claridad

5. Documentación de Referencia: para saber sobre que se basa

6. Normativa de Referencia Adoptada: reconocidas internacionalmente por el tipo de Industria

7. Marco Legal (indicar ámbito de aplicabilidad)

8. Listado de Mejores Prácticas

9. Anexos: alguno de los cuales pueden ser:

- Criterios de Aceptación y Rechazo

- Análisis FMEA de posibles Fallas

- Planilla de Responsables y Frecuencias de Realización de las Tareas Rutinarias a Ejecutar

- Planilla de Estimado Típico de Costos de Insumos
- Planilla de definición de Condición Básica

Esto es sumamente importante y requiere su tiempo para realizarse, pero una vez que se tiene, toda la compañía sabe cómo encarar acciones sobre los equipos de la misma forma.

Este si bien parece una Documentación estática puede considerarse dinámica pero lógicamente requiere la participación y ordenamiento estipulado desde su creación.

Esto da como resultado un accionar basado en el análisis, da confiabilidad y da la posibilidad de fijar objetivos con mayor precisión frente a la competencia.

\section{e) Análisis de Confiabilidad}

En este punto se trata de evaluar la capacidad de registrar los acontecimientos, su análisis tratando de encontrar causas frente a la necesidad de reparación, las mejoras logradas con la aplicación de los diferentes tipo de mantenimiento, las estadísticas e índices de gestión, la identificación de los equipos críticos y su priorización en la operación y sobre todo en el Mantenimiento, el ordenamiento de las Ordenes de Trabajo, su planificación y Programación y la confiabilidad de la acción de Mantenimiento.

Si bien no es una visión total sobre lo observado, permite ingerir que existe una tendencia a trabajar ordenado por los sectores que se pudo ver, con criterios de sistematización en los mismos, por convencimiento propio o quizás también, por exigencia del sistema del cliente, aunque no se pudo vislumbrar una unicidad de 
criterios en la forma de llevar a delante los registros, identificar las tareas, codificaciones comunes, acciones de análisis con criterios universales, sino mas bien la buena experiencia aplicada en el punto de acción y no siempre compartida o conocida por el resto, producto de la falta quizás de un sistema integrador. Esto hace perder muchas energías y no captura la riqueza de la experiencia del personal frente a distintas situaciones.

De la evaluación un total de 25,58 puntos de promedio sobre 40 posibles, se muestra una más que interesante perspectiva de trabajo en este campo.

Esto se logra con buenas bases de datos, registros confiables, buenos análisis de riesgo, RCM y Análisis de causa raíz.

\section{f) Medidas de Desempeño y Bench- marking}

Si bien en este eje se involucran dos áreas, que parecen disímiles, están directamente vinculadas a través que una genera los indicadores que utilizará y la otra realiza un análisis del estado de situación.

In situ, se pudo observar que en general hay dos tipos de situaciones que muestran muy claramente el estado de situación independientemente la autoevaluación:

1. Los indicadores estaban establecidos por la aplicación de las Normas implementadas ISO y OSHA. Esto esta muy claro, existía un seguimiento y preocupación explicita y se puede utilizar perfectamente para el Benchmarking.
2. Los indicadores tradicionales exigidos por los contratos con los clientes se llevaban en forma "particular" de acuerdo al contrato. Si bien existían indicadores comparables como porcentaje de Preventivo realizado, porcentaje de tareas programadas, etc., no era suficiente para decir que se tiene un ordenamiento reconocido por otras empresas aunque si por cada Grupo empresario particular como el caso de una importante Petrolera Internacionalmente reconocida, cliente de la Empresa.

Por todo lo expuesto, se sugirió la aplicación de indicadores según lo expresado en el ANEXO E de la norma ISO 142242006 como referencia.

No obstante se puede comentar que la autoevaluación reflejó un puntaje de 24.67 sobre 48 posibles es decir $51 \%$ de percepción de estas necesidades por parte de los distintos sectores que seguramente individualmente consideraban la importancia de estos rubros por lo que hay una interesante posibilidad de trabajo en esta área.

\section{g) Tecnología de Información}

En este eje se destaca lo observado y lo autoevaluado, quedando como recomendación establecer un sistema único para trabajar en todos los emprendimientos y que contemple una base de datos integral que pueda ser consultada por todos los integrantes que la necesiten con su régimen de autorizaciones correspondiente pero que permita a los usuarios tener 
referencias entre sí y utilizar lo que se va registrando.

Indudablemente, esto requiere una serie de pasos que estratégicamente se deberán dar como son:

1. Crear un sistema de gestión que pueda ser utilizado por todos.

2. Establecer las redes de comunicación necesarias para realizar lo expresado en 1.

3. Dar participación en la creación a los usuarios.

4. Establecer un interfaz con los sistemas corrientes de los clientes. Por ejemplo SAP, MAXIMO, etc.

La base de este sistema debe ser sin lugar a duda el trabajo ya comenzado por el personal con la Norma ISO 14224 lo que permite la estandarización.

No obstante se detectó muy buena predisposición en este sentido, esfuerzos y algunas concreciones orientadas en este sentido. Este punto no se limita solamente al sistema de gestión sino a pensar en otras alternativas como la Telemática y los Sistemas Expertos que seguramente serán factores diferenciadores en el mediano plazo.

La autoevaluación reflejó un 16.25 de puntaje sobre 44 posibles lo que sin duda marca uno de los ejes más débiles en la visión del personal.

\section{h) Planificación y Programación de Trabajos}

Si bien se puede trabajar en este aspecto, indudablemente es uno de los puntos percibidos como más fuertes dentro de la Organización. Es una fortaleza de la misma. No obstante se permite sugerir que esta es un área de mejora sustancial. Para ello se deberá integrar la experiencia del capital de conocimientos, ordenamiento de los mismos, distribución en los distintos niveles y adecuación a su utilización como lenguaje y modo de operar común para toda la organización.

Se refiere a que en esta parte, los PROCESOS y las TÁCTICAS son esenciales y requieren una definición estratégica para poder realizarlas.

La visión del personal en esta área es alta de 29.92 puntos sobre 44 lo que marca un $68 \%$ interesante y refleja claramente lo observado en el campo de acción.

\section{i) Administración de repuestos y ma- teriales}

Este punto se refiere la autoevaluación en la que sobre 48 puntos posibles el promedio arrojó 21.17 puntos o sea un $44 \%$.

Es importante destacar que solo se tuvo algunas charlas sobre el tema con la Gerencia y con algún de los Responsables que induce a sugerir que este es uno de los PROCESO críticos que deben ser definidos y puesto en funcionamiento en forma integral.

La prestación de repuestos para los distintos emprendimientos esta en general reservada al cliente aunque algunos ítems como así también insumos son provistos por el contratista lo cual lleva a pensar que es necesario una Ingeniería de Materiales que debe ser aplicada, en la que se debe considerar, disponibilidad, calidad, 


\section{ReLAInEP}

precio y uniformidad de criterios basados en la experiencias y know-how de la Empresa.

En este rubro se detectan importantes recursos económicos en juego y se requiere una inversión para ser aprovechados eficientemente, inclusive, como factor de Benchmarking.

\section{j) Reingeniería de Procesos de Mante- nimiento}

Según lo expresado anteriormente, el PROCESO y, mas específicamente aplicado al área Mantenimiento, es esencial y define el QUE, por lo que indirectamente tiene que ver con Calidad, Seguridad, Costos, Tiempos, Oportunidad de Negocio, Competencia, Valor Agregado y Mejora Continua.

Elemento dinámico, que requiere experiencia y tecnología, pero sobre todo participación, comunicación e involucramiento de las distintas áreas.

La autoevaluación refiere sobre 28 puntos po- sibles indica un promedio de 9.75 puntos lo cual alcanza al menos un sentido de conocimiento y /o participación del $35 \%$, cifra por demás reveladora de un posibilidad de trabajo por demás interesante y necesaria.

De los Procesos nacerán los Procedimientos, las Tácticas y las Indicaciones Operativas que alimentarán referencialmente a los RR.HH., valor indiscutible de la Empresa. De ahí su importancia.

\section{k) Diagrama de situación definido por autoevaluación}

Resumiendo todos los puntos anteriores se puede graficar los diferentes ejes considerados todos juntos estableciendo un diagrama (Figura 1) referencial que permite saber el estado de situación relevado por la encuesta de autoevaluación y a partir de ahí generar un plan de acción para el futuro.

\section{FIGURA 1: DIAGRAMA DE AUTOEVALUACIÓN}

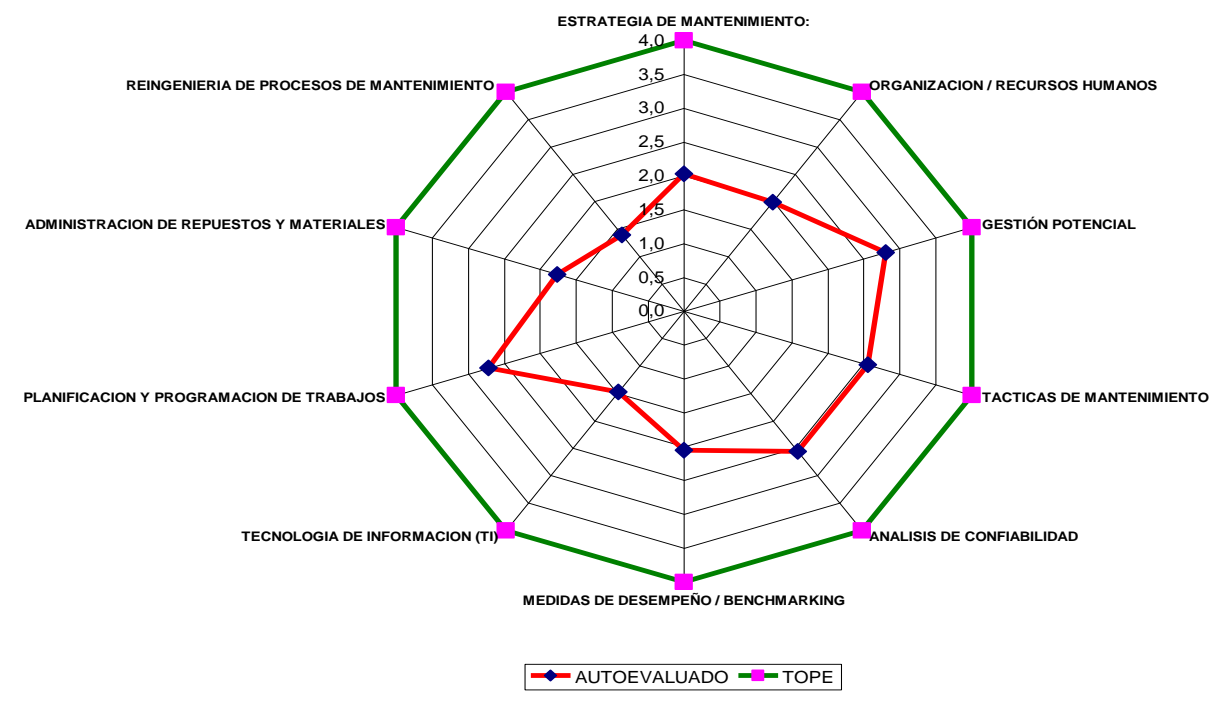


Los alcances de las acciones a proponer están directamente relacionadas con el plan estratégico que se desee implementar, aunque se pueden visualizar acciones potenciales en cada uno de los ejes relevados.

\subsection{Análisis de resultados relevados}

Sobre la base de la información relevada y no perdiendo de vista el objetivo inicial de "Mostrar una Gestión ordenada y en proceso de mejora continua" y con una visión de Empresa que trabaja bajo Normas Internacionales reconocidas, como ISO 14224 por ejemplo, se puede expresar lo siguiente:

La empresa contaba con una Gestión de Operación y Mantenimiento en vigencia respaldada por los diferentes clientes con que cuenta.

Los RR.HH. tenían una experiencia más que interesante y un potencial muy importante verificable por las intervenciones durante la capacitación realizada y la posterior vinculación en el comienzo de la implantación de la Norma ISO 14224.

Se verifican operaciones muy bien realizadas, controladas e informadas en áreas como lo referido a la Norma ISO 9001-2000 de Calidad, la ISO 14001 referida a Medio Ambiente y la OSHA 18001 de gestión del RR.HH.

Se muestran sistemas de gestión de Mantenimiento generados por propio personal de la Empresa. La motivación a la mejora es un punto importante que se vió en algunos integrantes.

La Misión y Visión de la Empresa es clara aunque debería tener una estructura más diná- mica e ir adaptándose sobre la base de un Plan Estratégico, elemento que supone existe aunque no se pudo verificar.

Los Valores definidos se vislumbran aunque no se tiene la seguridad que todos lo conozcan como lo referido al punto anterior.

Están dadas las condiciones y de hecho ya se comenzó para el desarrollo e implementación de la NORMA ISO 14224 lo cual permite unificar criterios, establecer un lenguaje común y tener un sistema de Gestión de Activos de los distintos clientes integrado para poder utilizarse en los emprendimientos actuales y con los futuros clientes. Hasta donde se pudo ver no se vislumbraba una política de desarrollo de capacidades de RR.HH. adecuada a las necesidades de la Empresa.

Los integrantes con los que se tuvo oportunidad de vincularse, demuestran conocimientos del negocio del Mantenimiento de Industrias Petroleras y las necesidades y carencias que se tienen, suplidas muchas veces con empuje y experiencia, aunque sin una metodología que apunte a la productividad y más aún generar los elementos para mostrar a futuros clientes.

El lenguaje común es un factor decisivo, para no trabajar en forma reactiva frente a la necesidad del cliente, sino posicionarse en una visión y ejecución proactiva de donde saldrá el valor agregado.

\section{Propuestas de acción para la aplica- ción en la Industria}

Una vez realizada la autoevaluación, relevado los datos in-situ y habiendo hecho un análisis de situación se propone: 
- Continuar con la implementación piloto de la Norma ISO 14224 sobre la base de identificar un sistema, con sus componentes, realizar simultáneamente la Identificación, registro y sistematización de fallas y parámetros de Mantenimiento sobre dos situaciones: las potenciales que se vayan analizando y las reales que se sucedan, conformando un árbol de equipos y un registro de fallas que permita tomar acciones comunes, definir los tipos de mantenimientos que se deban realizar, las mejoras a implementar y armar una gran base de datos que alimente un Sistema de Gestión para referencia de los integrantes de la Empresa que deban nutrirse de él para el trabajo diario.

- Evaluar los resultados obtenidos y las correcciones a realizar.

- Extender la Implantación al resto del equipamiento.

- Proponer a las Direcciones y Gerencias la identificación sobre el perfil de Empresa que se quiere tener en el mediano y largo plazo sobre la base de la autoevaluación detallada arriba y conforme a los siguientes ejes estratégicos conceptuales:

\section{Estrategia}

Administración De RR.HH.

Planificación y Programación

Tácticas de Mantenimiento

Indicadores de Perfomance

Información Tecnológica

\section{Análisis de Confiabilidad \\ Involucramiento del personal \\ Procesos}

Proponer la generación desde Sistemas de un acceso fácil, ordenado y amigable mediante un sistema de gestión a todos los registros e información que se vaya generando. Integrar el proceso propuesto con lo existente en Seguridad, Calidad y Medio Ambiente.

Avanzar en la Generación de Procesos entendidos como: conjunto de actividades mutuamente relacionadas o que interactúan y transforman elementos de entrada en resultados previamente definidos, o sea algo que se ejecuta repetidamente y tiene que ver con la organización y cruza la misma en todas las direcciones: tanto verticalmente como horizontalmente, definiendo que nunca una sola área departamental debería ser dueño de todo un proceso, aunque pueda ser una gran parte del mismo, pero no el $100 \%$.

Identificar los requerimientos de datos típicos de los distintos equipos con se le solicitarán a los clientes y con los cuales se trabajará.

Identificar los indicadores de gestión y benchmarking.

Confeccionar Tácticas de Equipo comunes como referencia del Know-how de la Empresa, reconocido por todos para la realización de tareas de Mantenimiento, entendiendo por TACTICAS lo expresado mas arriba.

Preparar la implementación de acciones para el mediano y largo plazo sobre la base del siguiente modelo Gestión Integral de Activos.

El modelo propuesto se basa en una pirámide 


\section{ReLAlnEP}

(Figura 2) donde la base es un fuerte liderazgo con acento en el Management y la Estrategia de Negocio.

En todo proceso el Control es fundamental y constituye la segunda parte de la pirámide de gestión. En la misma se puso atención en la Administración y Manejo de los Datos, la elaboración de Tácticas, la Administración y Manejo de los Materiales, la Planificación, Programación y Medición como pautas a seguir.

\section{FIGURA 2: MODELO PROPUESTO}

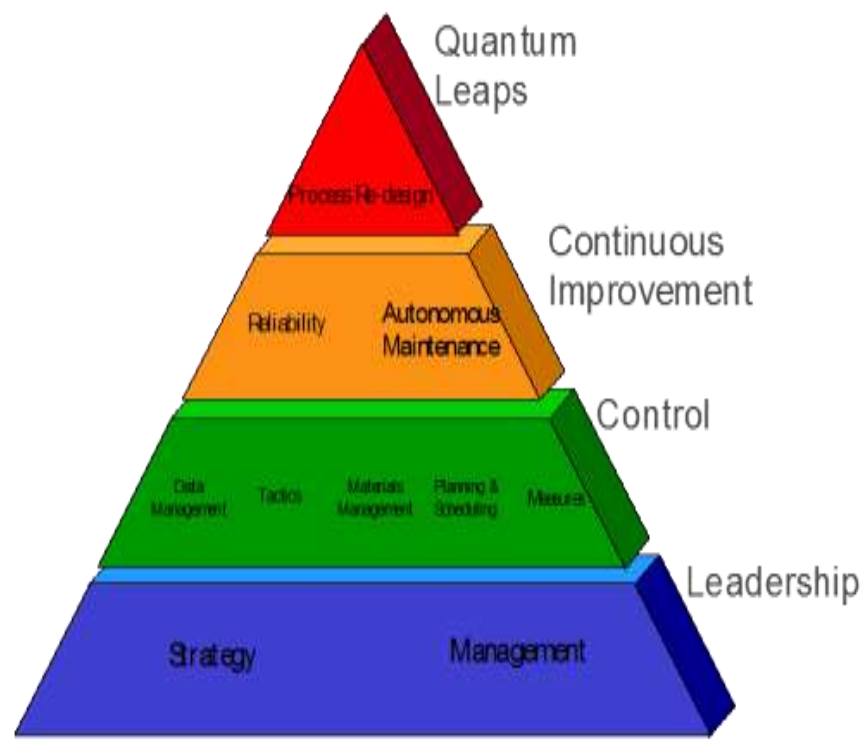

Las mejoras en la Confiabilidad y el Mantenimiento Autónomo son esenciales como paso posterior para inducir el proceso de mejora continua, terminando en la cresta de la pirámide con el Rediseño de los Procesos.

\section{Conclusiones}

Se arribó a las siguientes conclusiones luego del desarrollo de trabajo mostrando los resultados obtenidos y los que se pueden lograr:

La Empresa tenía una serie de procesos y procedimientos reconocidos en el área Seguridad, Medio Ambiente y Calidad que la posicionaban claramente en competencia y capacidad de liderar a las compañías de servicios hidrocarburíferos y afines.

Se contaba con los recursos humanos orientados en el sentido expresado en el punto anterior.

Por otro lado se comenzó un proceso de alineamiento con la Norma ISO 14224 para sus emprendimientos.

Se debe confeccionar Procesos básicos y reconocidos por todos sus RR.HH que involucren a varias áreas y las vinculen entre sí, referidas al manejo de las Tareas de Mantenimiento y sus recursos, como por ejemplo Planificación, Desarrollo de RR.HH. Gestión de Materiales, etc. Se recomendó que el número no fuera 


\section{ReLAInEF}

superior a 8 por una cuestión de conocimiento y manejo.

Se propuso Tácticas para los diferentes tipos de equipos comunes a varias plantas, que definan el Know-how de Empresa, para realizar las tareas de mantenimiento, con la participación de todos los involucrados.
Se recomendó definir el Plan Estratégico para sus próximos años en función de cómo querer posicionarse en el mercado en el que actúa.

Por último se propuso modelo gráfico a modo de resumen como visión para poder mostrar a la Empresa posicionada frente a los clientes actuales y futuros donde se establecieron las siguientes pautas (Figura 3).

FIGURA 3: PAUTAS FUTURAS

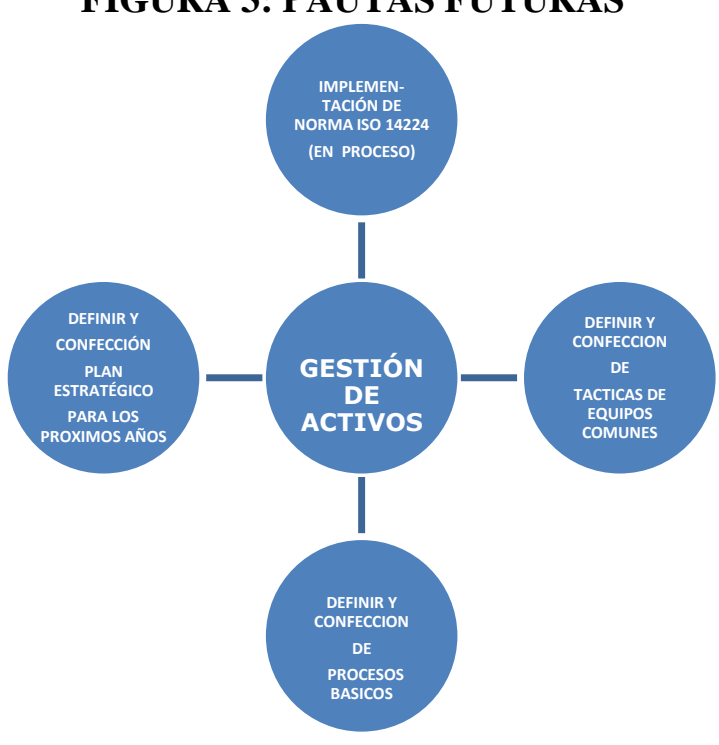

\section{Referências}

MORROW, L.C. Manual de mantenimiento industrial (3 tomos). Compañía editorial continental s.a., México, 1974.

PISTARELLI, A.J. Manual de mantenimiento: ingeniería, gestión y organización, editorial: Alejandro Jorge Pistarelli, República Argentina, 696p, 2010

MORA GUTIERREZ, A. Mantenimientoplaneación, ejecución y Control. ALFA OMEGA GRUPO EDITOR, ESPAÑA, 2010.

INTERNATIONAL ORGANIZATION FOR STANDARDIZATION. ISO 14224-2006. Petroleum, petrochemical and natural gas industries- Collection and Exchange for reability and maintenance data for equipment, 2010.
FURLANETTO L.; ARATA, A. Manual de Gestión de Activos y Mantenimiento. Ril Editores, República de Chile, paginas 924, 2005.

TAVARES, L. Administración moderna del Mantenimiento. Mantenimiento Mundial, Brasil, 2010.

INSTITUTE OF ASSET MANAGEMENT. Norma PASS 55-2:2008, Parte 1: Especificaciones para la gestión optimizada de activos físicos, España, 38p, 2009.

INSTITUTE OF ASSET MANAGEMENT. Norma PASS 55-2:2008, Parte 2: Directrices para la aplicación de PASS 55-1, España, páginas 71, 2009. 